\title{
Extração Mista e Transesterificação Metílica de Óleo de Syagrus Romanzoffiana ${ }^{1}$
}

Josélia Dobrzanski² ${ }^{2}$ Alan Gabriel Adamczevski ${ }^{3}$, Sandra Regina Masetto Antunes ${ }^{4}$, Eriel Biagini Sabino ${ }^{5}$, Maria Elena Payret Arrua ${ }^{4}$, Augusto Celso Antunes ${ }^{4}$, Pedro Henrique Weirich Neto ${ }^{4}$

1 Aceito para publicação no $3^{\circ}$ Trimestre de 2014

2 Mestranda em Bioenergia na Universidade Estadual de Ponta Grossa, jomly_ds@hotmail.com

3 Mestrando em Química Aplicada na Universidade Estadual de Ponta Grosa, adamczevski@gmail.com

${ }^{4}$ Professores do Programa de Pós- Graduação em Bioenergia na Universidade Estadual de Ponta Grossa, sandrareg@uepg.br; elenpayret@gmail.com; ac_antunes@uol.com.br; lama1@uepg.br

5 Técnico na Universidade Estadual de Ponta Grossa no Departamento de Física, erialsab@yahoo.com.br

\section{Resumo}

O Brasil dispõe de uma grande variedade de espécies vegetais que se podem extrair óleos para produção de biodiesel. A espécie Syagrus romanzoffiana se apresenta como uma matéria prima promissora e alternativa na produção de biocombustíveis. Esta é uma palmeira presente em diversas regiões do Brasil, da família Arecaceae, comumente conhecida como jerivá e produz frutos o ano todo, com alto teor de óleo. $\mathrm{O}$ presente estudo objetivou avaliar o sistema misto de extração (mecânica e solvente) do óleo de $S$. romanzoffiana, verificar as propriedades físico-químicas e utilizá-lo na produção de biodiesel. Após a extração, o óleo foi avaliado em relação ao rendimento e caracterizado pelos ensaios de massa específica, viscosidade, estabilidade oxidativa e RMN de ${ }^{1} \mathrm{H}$. O rendimento obtido por extração mista foi de $57 \% \mathrm{~m} / \mathrm{m}$. Pela análise do produto da transesterificação por $\mathrm{RMN}$ de ${ }^{1} \mathrm{H}$ verificou-se uma elevada taxa de conversão dos ésteres.

Palavras-chave: Syagrus romanzoffiana, biocombustíveis, extração mista 


\begin{abstract}
Brazil has a variety of plant species that can extract oils for biodiesel production. The Syagrus romanzoffiana species is presented as a promising alternative feedstock and the production of biofuels. This is present in several regions of Brazil, Arecaceae family, commonly known as jerivá palm tree, which produces fruit all year round, with high oil content. The present study aimed to evaluate the system of mixed extraction, mechanical and solvent extraction of $S$. romanzoffiana oil, check the physicochemical properties and use it in the production of biodiesel. After extraction, the oil was evaluated in relation to the yield and characterized by testing specific gravity, viscosity, oxidative stability and ${ }^{1} \mathrm{H}$ NMR. The yield obtained by mixed extraction was $57 \% \mathrm{~m} / \mathrm{m}$. The transesterification product analysis by ${ }^{1} \mathrm{H} \mathrm{NMR}$, there was a high rate of conversion of esters.
\end{abstract}

Keywords: Syagrus romanzoffiana, biofuels, mixed extraction

\title{
Introdução
}

O Brasil dispõe de uma grande variedade de espécies vegetais oleaginosas para produção de biodiesel, como soja, algodão, milho, girasol, palma, entre outras (JANSSEN e RUTZ, 2011; RATHMANN et al., 2012) . Outras espécies oleaginosas alternativas podem ser promissoras para produção de biodiesel. Dentre estas espécies a Syagrus romanzoffiana se apresenta como uma matéria prima promissora (MOREIRA, et al. 2013). Esta palmeira comumente denominada de jerivá pertence à família Arecaceae, sendo encontrada facilmente em diversas regiões do Brasil e produz frutos o ano todo. Os frutos são do tipo drupa, globosos ou ovóides, de cor amarela ou alaranjada, com endocarpo-rígido, que envolve uma amêndoa oleosa e um mesocarpo fibroso, suculento e adocicado (LORENZI, 2010).

De acordo com Moreira et al. (2013) as amêndoas destes frutos apresentam um teor de óleo de $52 \% \mathrm{~m} / \mathrm{m}$, sendo $71 \%$ do mesmo, composto de ácidos graxos saturados. Entretanto, o rendimento de óleo está diretamente relacionado com o método de extração escolhido.

Revista Brasileira de Energias Renováveis, v. 3, p.299- 305, 2014 
Os métodos básicos mais comuns de extração de óleos são a prensa hidráulica por batelada, prensa mecânica contínua e extração por solventes (WAKELYN e PETER, 2005). A prensagem mecânica, geralmente, é efetuada por prensas contínuas, que são usadas para uma parcial remoção de óleo, seguida por extração com o solvente, constituindo o processo misto. O objetivo deste trabalho foi realizar a extração de óleo de $S$. romanzoffiana por sistema misto e a produção de biodiesel por transesterificação metílica.

\section{Materiais e métodos}

Primeiramente foi projetado e confeccionado um molde para prensagem mecânica uniaxial das amêndoas de S. romanzoffianna (Figura 1).

(a)

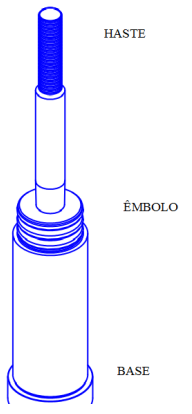

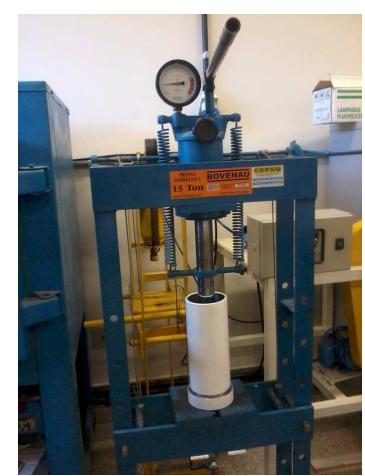

(b)

Figura 1: a) Molde elaborado para prensagem das amêndoas do $S$. romanzoffianna. b) Prensa hidráulica utilizada no processo de extração

Para a extração mecânica foi utilizada uma prensa hidráulica uniaxial. A amostra de óleo obtida foi coletada diretamente do sistema e submetida à filtração para eliminação de eventuais partículas sólidas. Após a coleta, a torta resultante foi submetida à extração contínua com aparelho de Soxhlet utilizando $n$-hexano como solvente, durante $6 \mathrm{~h}$.

As propriedades físico-químicas do óleo obtido foram determinadas com relação à massa específica, viscosidade, índice de acidez e estabilidade oxidativa. Para a determinação da estabilidade oxidativa foi utilizado um equipamento Rancimat Methrohm, modelo 893, de acordo com EN 14112:2003. A determinação da massa específica foi realizada utilizando um microviscosímetro digital da marca Anton Paar modelo Lovis 200 ME.

Revista Brasileira de Energias Renováveis, v. 3, p.299- 305, 2014 
O óleo foi também caracterizado por RMN de ${ }^{1} \mathrm{H}$, em um equipamento BRUKER $\operatorname{ASCEND}^{\mathrm{TM}}$ 400. Após a caracterização do óleo o mesmo foi submetido ao processo de transesterificação com metanol e catalisador homogêneo NaOH (FERRARI; OLIVEIRA; SCABIO, 2005). A reação foi acompanhada por cromatografia em camada delgada com o uso de padrões e por RMN de ${ }^{1} \mathrm{H}$. Todas as análises de $\mathrm{RMN}$ de ${ }^{1} \mathrm{H}$ foram realizadas em $\mathrm{CDCl}_{3}$.

\section{Resultados e discussão}

O rendimento total de óleo obtido, pela extração mista, foi de $57 \% \mathrm{~m} / \mathrm{m}$. Este valor, que expressa o conteúdo de óleo nas amêndoas do $S$. romanzoffianna, é bastante significativo se comparado com o teor de óleo de outras espécies oleaginosas como amêndoas de dendê (26\%), sementes de girassol (38-48\%), colza (40-48\%), mamona (43-45\%), amendoim (40$50 \%$ ) e soja (17\%) entre outras (BIODIESELBR, 2014).

Na Tabela 1 são mostrados os valores das propriedades físico-químicas do óleo de $S$. romanzoffianna que expressam a qualidade do mesmo.

Tabela 1: Parâmetros de qualidade do óleo de amêndoas do S. romanzoffianna

\begin{tabular}{lll}
\hline Parâmetros & Óleo (extração mecânica) & Óleo (extração da torta) \\
\hline $\begin{array}{l}\text { Massa específica a } 20^{\circ} \mathrm{C} \\
\left(\mathrm{kg} \mathrm{m}^{-3}\right)\end{array}$ & $920 \pm 7$ & $900 \pm 6$ \\
\hline Viscosidade Cinemática & $32,9 \pm 0,05$ & $33,4 \pm 0,05$ \\
à $40^{\circ} \mathrm{C}\left(\mathrm{mm}^{2} \mathrm{~s}\right)$ & \\
\hline Estabilidade Oxidativa & $23 \pm 0,4$ & $23 \pm 0,9$ \\
à $110^{\circ} \mathrm{C}$ (horas) & \\
\hline Índice de Acidez & $1,45 \pm 0,04$ & $1,55 \pm 0,03$ \\
(mg KOH g $\left.{ }^{-1}\right)$ & & \\
\hline
\end{tabular}

Os valores determinados de massa específica e viscosidade cinemática (Tabela 1) são comparáveis aos encontrados na literatura para óleo de $S$. romanzoffianna e para óleo de soja (MOREIRA, et al., 2013).

Observam-se na Tabela 1 elevados valores de estabilidade oxidativa, o que indica o favorecimento da estocagem do óleo de $S$. romanzoffiana e, baixo índice de acidez o que é 
favorável para a reação de transesterificação, sem prévio tratamento.

O óleo de S. romanzoffiana também foi caracterizado por RMN de ${ }^{1} \mathrm{H}$, Figura 2.

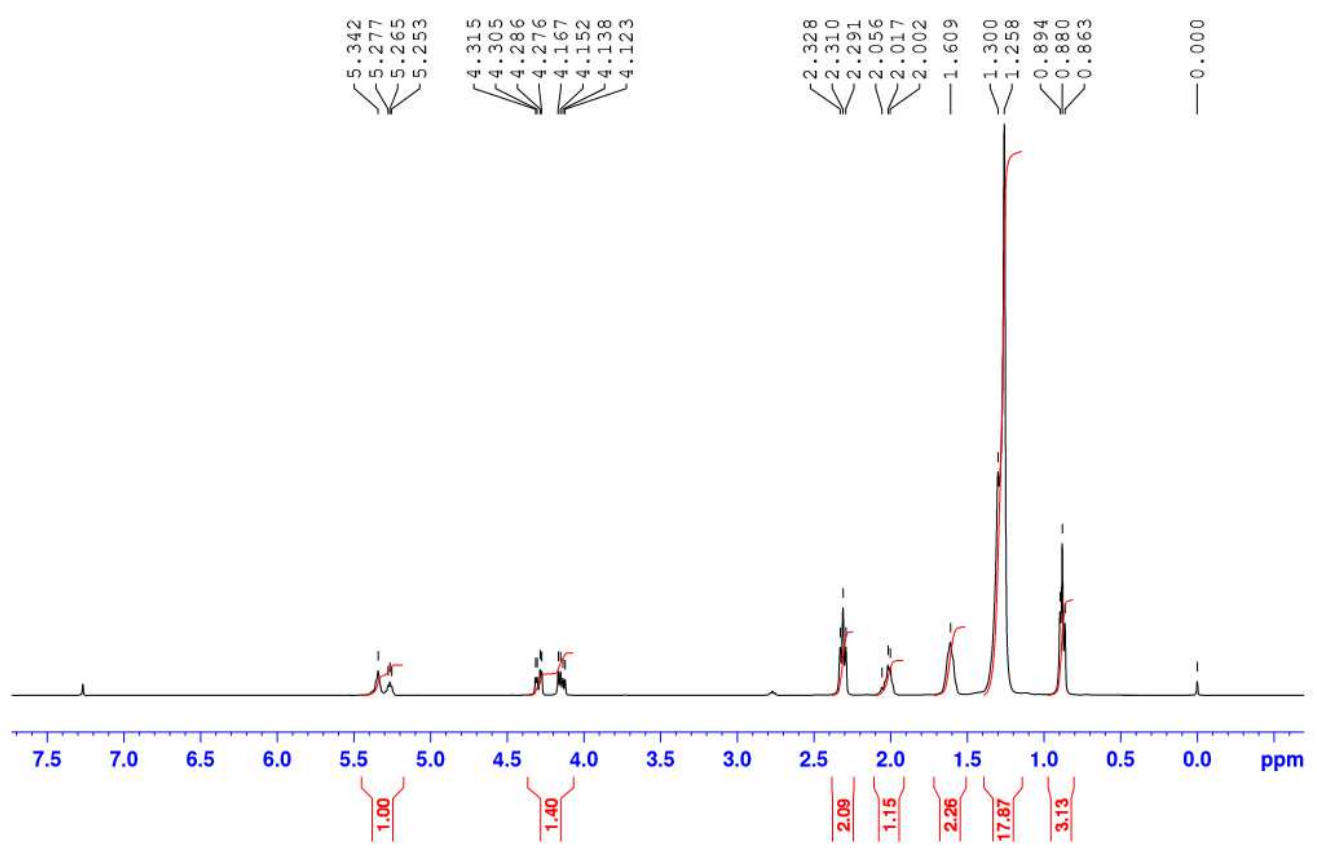

Figura 2: Espectro de $\mathbf{R M N}$ de ${ }^{1} \mathrm{H}$ do óleo de $S$. romanzoffiana

Pela análise do espectro de $\mathrm{RMN}$ de ${ }^{1} \mathrm{H}$ do óleo de $S$. romanzoffiana da Figura 2, podem ser observados os sinais correspondentes ao triacilglicerídeo, sendo que, a cadeia preponderantemente encontrada corresponde ao ácido oleico (C18:1). Pelo espectro da Figura 2 observa-se um multipleto na região de $\delta(5,38-5,34)$ ppm que corresponde aos hidrogênios $\mathrm{H}$ vinílicos da porção oléica do óleo. Já na região de $\delta(5,29$ - 5,25) ppm observa-se outro multipleto correspondente ao $\mathrm{H}$ denominado como glicerólico. A presença dos hidrogênios $\alpha$ acílicos pode ser observada como um conjunto de sinais característicos (duplos dubletos, dd)

Revista Brasileira de Energias Renováveis, v. 3, p.299- 305, 2014 
em $\delta(4,31-4,12)$ ppm (JIN, et al., 2007). Os hidrogênios $H$ ( $\alpha$-carbonílicos) podem ser localizados em $\delta 2,01 \mathrm{ppm}$ como tripleto. Os sinais de hidrogênios metilênicos podem ser observados como multipleto na faixa de $\delta(1,42-1,1)$ e o sinal dos hidrogênios metílicos em $\delta$ 0,99 ppm como tripleto.

Pela análise do espectro da $\mathrm{RMN}$ de ${ }^{1} \mathrm{H}$, do produto da reação de transesterificação do óleo de Syagrus romanzoffiana, observou-se o desaparecimento dos sinais dos $\mathrm{H}$ acílicos (presentes na estrutura do óleo), o que indica a total conversão em ésteres metílicos.

\section{Conclusões}

O teor de óleo de S. romanzoffiana de $57 \% \mathrm{~m} / \mathrm{m}$ pode se considerado um valor elevado, quando comparado com outras espécies vegetais. Os parâmetros físico-químicos determinados e a conversão total em ésteres metílicos indicam que a $S$. romanzoffiana se apresenta como uma matéria-prima alternativa promissora para a produção de biodiesel.

\section{Agradecimentos}

CAPES (Coordenação de Aperfeiçoamento de Pessoal de Nível Superior), Fundação Araucária e C-LABMU (Complexo de Laboratório de Multiusuários da UEPG).

\section{Referências}

BIODIESELBR. Rendimento de óleo das Sementes. Disponível em: <http://www.biodieselbr.com/biodiesel/sementes/rendimento-sementes-oleo.htm> Acessado em 03 set. 2014.

FERRARI, R. A.; OLIVEIRA, V. da S.; SCABIO, A. Oxidative stability of biodiesel from soybean oil fatty acid ethyl esters. Scientia Agricola, vol. 62, n.3, p. 291-295, 2005.

JANSSEN, R., RUTZ, D.D. Sustainability of biofuels in Latin America: risks and opportunities. Energy Policy 39, 5717-5725, 2011. 
JIN, F.; KAWASAKI, K.; KISHIDA, H.; TOHJI, K.; MORIYA, T.; ENOMOTO, H. NMR Spectroscopic study on methanolysis reaction of vegetable oil. Fuel, vol. 86, n7-8, p. 12011207, 2007.

LORENZI, H., KAHN, F., NOBLICK, L. R., FERREIRA, E. FLORA BRASILEIRA ARECACEAE (PALMEIRAS), vol. 347. Instituto Plantarum, Nova Odessa, SP. 2010.

MOREIRA, M. A. C.; ARRÚA, M. E. P.; ANTUNES, A. C.; FIUZA, T. E. R.; COSTA, B. J. WEIRICH NETO, P. H. ANTUNES, S. R. M. Characterization of Syagrus romanzoffiana oil aiming at biodiesel production. Industrial Crops \& Products. p. 57-60, 2013.

RATHMANN, R., SZKLO, A., SCHAEFFER, R. Targets and results of the Brazilian biodiesel incentive program - has it reached the promised land? Appl. Energy 97, 91-100, 2012.

WAKELYN, P. J.; PETER, J. W. Solvent Extraction to Obtain Edible Oil Products. Handbook of Functional Lipids. Casimiro C. Akoh (Ed). ISBN 978-0-8493-2162-7. p. 89$131,2005$. 